\title{
New wave behaviors of the (3+1)-dimensional Date-Jimbo-Kashiwara-Miwa equation
}

\author{
S. Şule Şener Kılıç ${ }^{1}$ \\ ${ }^{1}$ Department of mathematics, Faculty of Science, Ataturk University, 25240, Erzurum, Turkey
}

\section{Article Info}

Keywords: $(3+1)$-dimensional DateJimbo-Kashiwara-Miwa equation, generalized exponential rational function method, exact solutions

2010 AMS: 35Q99, 35Q92.

Received: 11 November 2021

Accepted: 21 December 2021

Available online: 29 December 2021

\begin{abstract}
In this study, the $(3+1)$-dimensional Date-Jimbo-Kashiwara-Miwa equation that indicated the propagation of nonlinear dispersive waves in inhomogeneous media is given for consideration. The generalized exponential rational function method is used to seek some new exact solutions for the considered equation. The three-dimensional surfaces and twodimensional graphs of the obtained solutions are plotted by choosing the appropriate values of the involving free parameters.
\end{abstract}

\section{Introduction}

Nonlinear partial differential equations (NPDEs) are frequently employed to subedit real-world issues from a mathematical standpoint. There are several situations in which simulations of these instances are necessary. These important areas include fiber optics, fluid mechanics, electromagnetic theory, plasma physics, nuclear physics, mass transport, hydrodynamics, population and economics, and many more [1-10]. As a consequence, exact analytic solutions to such equations become the most important factor to consider, because they can supply important data for accurate explanations of natural processes. Many academics and mathematicians have worked hard over the last few decades to find explicit solutions to NPDEs. For this aim, many analytical methods have been created to obtain such exact solutions like sin-Gordon expansion method [11-14], the (1/ $\left.G^{\prime}\right)$-expansion method [15-17], the simplified Hirota's method [18-20], the Backlund transformation method [21,22], and symbolic computational method [23].

The (2+1)-dimensional Date-Jimbo-Kashiwara-Miwa (DJKM) reads [24-26]

$$
Q_{x x x y}+4 Q_{x x y} Q_{x}+2 Q_{x x x} Q_{y}+6 Q_{x y} Q_{x x}-\alpha Q_{y y y}-2 \beta Q_{x x t}=0
$$

where $\alpha$ and $\beta$ are real constants, $Q(x, y, t)$ indicated the wave's maximum extension as evaluated from its equilibrium state. Eq. (1.1) represents the propagation of nonlinear dispersive waves in inhomogeneous media. The DJKM equation was first developed by Date et al. [24]. In [27], Wazwaz has developed the (2+1) dimensional DJKM equation to a new (3+1)-dimensional DJKM equation by adding the term $\left(k Q_{x}+r Q_{y}+s Q_{z}\right)_{x x}$ which is given by

$$
Q_{x x x y}+4 Q_{x x y} Q_{x}+2 Q_{x x x} Q_{y}+6 Q_{x y} Q_{x x}-\alpha Q_{y y y}-2 \beta Q_{x x t}+\left(k Q_{x}+r Q_{y}+s Q_{z}\right)_{x x}=0 .
$$

Wazwaz demonstrated that Eq. (1.2) is a completely integrable equation via using the Painlevé test, and used Hirota's simple method to construct multiple soliton solutions as well as solitonic, singular, and periodic solutions. In [28], the authors used three analytical methods namely the $\exp (-\phi(\xi))$ - expansion method, the $\left(G^{\prime} / G\right)$ - expansion method, and the sine-Gordon expansion method to Eq. (1.2), as a result, several exact solutions have been obtained. Furthermore, they used the finite difference method to attain some numerical solutions. In this paper, we will use the generalized exponential rational function method (GERFM) to construct some new exact solutions to the (3+1)-dimensional DJKM equation. 


\section{Description of the method}

In this section, the basic concepts of the GERFM [29-32] are given:

Step 1: Consider the general form of NPDE as

$$
\Psi\left(Q(x, y, z, t), Q_{x}, Q_{y}, Q_{z}, Q_{t}, Q_{x t}, Q_{x x}, Q_{y t}, Q_{y y}, \ldots\right)=0 .
$$

Let us take the wave transformation as follows:

$$
Q(x, y, z, t)=\psi(\varsigma), \varsigma=a x+b y+d z-c t,
$$

where $a, b, d$, and $c$ are constants to be calculated. Using Eq. (2.2) into Eq. (2.1), we get the following nonlinear ordinary differential equation:

$$
\Theta\left(\psi, \psi^{\prime}, \psi^{\prime \prime}, \psi^{\prime \prime \prime}, \ldots\right)=0
$$

Step 2: Let the solution of Eq. (2.3) have the following form

$$
\psi(\varsigma)=a_{0}+\sum_{j=1}^{N} a_{j} \phi(\varsigma)^{j}+\sum_{j=1}^{N} b_{j} \phi(\varsigma)^{-j},
$$

where

$$
\phi(\varsigma)=\frac{\gamma_{1} e^{\lambda_{1} \varsigma}+\gamma_{2} e^{\lambda_{2} \varsigma}}{\gamma_{3} e^{\lambda_{3} \varsigma}+\gamma_{4} e^{\lambda_{4} \varsigma}},
$$

where $\lambda_{m}, \gamma_{m}(1 \leq m \leq 4)$ are real/complex constants, $a_{0}, a_{j}, b_{j}$ are constants to be calculated, $N$ will be found by balance principle. Step3: Inserting Eq. (2.5) in Eq. (2.4) then using Eq. (2.4) in Eq. (2.3), then we get a polynomial which is dependent on Eq. (2.5). By setting the like order terms equal to zero, we achieve a system of algebraic equations concerning $a, b, c, d, a_{0}, a_{1}$, and $b_{1}$. Solving the obtained system with the aid of any symbolic computation software, the values of the involved parameters will be determined and replacing the determined values into Eq. (2.3), one may obtain the solutions of Eq. (2.1).

\section{Applications of model}

In this section, we apply the GERFM to Eq.(1.2). Firstly, by inserting Eq. (2.2) into Eq.(1.2), we get

$$
6 a^{3} b \psi^{\prime \prime}(\varsigma)^{2}+\left(a^{3} k-b^{3} \alpha+a^{2}(b r+d s+2 c \beta)+6 a^{3} b \psi^{\prime}(\varsigma)\right) \psi^{\prime \prime \prime}(\varsigma)+a^{4} b \psi^{\prime \prime \prime \prime \prime \prime}(\varsigma)=0 .
$$

Inserting Eq. (3.1) twice, and set the constants of integration to zero, so one may get

$$
3 a^{3} b \psi^{\prime}(\varsigma)^{2}+\left(a^{3} k-b^{3} \alpha+a^{2}(b r+d s+2 c \beta) \psi^{\prime}(\varsigma)\right)+a^{4} b \psi^{\prime \prime \prime}(\varsigma)=0
$$

Using the balance principle between the term $\psi^{\prime \prime \prime}(\varsigma)$ and $\psi^{\prime}(\varsigma)^{2}$, we have $N=1$. Considering Eq. (2.4) with $N=1$, then it takes the below form

$$
\psi(\varsigma)=a_{0}+a_{1} \phi(\varsigma)+\frac{b_{1}}{\phi(\varsigma)}
$$

In what follows, different solitary wave solutions are constructed under certain conditions

Family 1: Using $\gamma=\{-2,-1,1,1\}, \lambda=\{0,1,0,1\}$ in Eq. (2.5), then one may have

$$
\phi(\varsigma)=\frac{-2-\mathrm{e}^{\varsigma}}{1+\mathrm{e}^{\varsigma}} .
$$

Case 1: $k=\frac{b^{3} \alpha}{a^{3}}, c=-\frac{b\left(a^{2}+r\right)+d s}{2 \beta}, a_{1}=2 a, b_{1}=0$.

$$
\psi_{1}(\varsigma)=a_{0}+\frac{2 a\left(-2-\mathrm{e}^{\varsigma}\right)}{1+\mathrm{e}^{\varsigma}}
$$

Then, one may have an exact solution of the exponential type

$$
Q_{1}(x, y, z, t)=a_{0}+\frac{2 a\left(-2-\mathrm{e}^{a x+b y+d z+\frac{\left(b\left(a^{2}+r\right)+d s\right) t}{2 \beta}}\right)}{1+\mathrm{e}^{a x+b y+d z+\frac{\left(b\left(a^{2}+r\right)+d s\right) t}{2 \beta}}} .
$$

Family 2: $\gamma=\{-2-i, 2-i,-1,1\}, \lambda=\{i,-i, i,-i\}$ into Eq. (2.5), then one may have

$$
\phi(\varsigma)=\frac{\cos (\varsigma)+2 \sin (\varsigma)}{\sin (\varsigma)}
$$



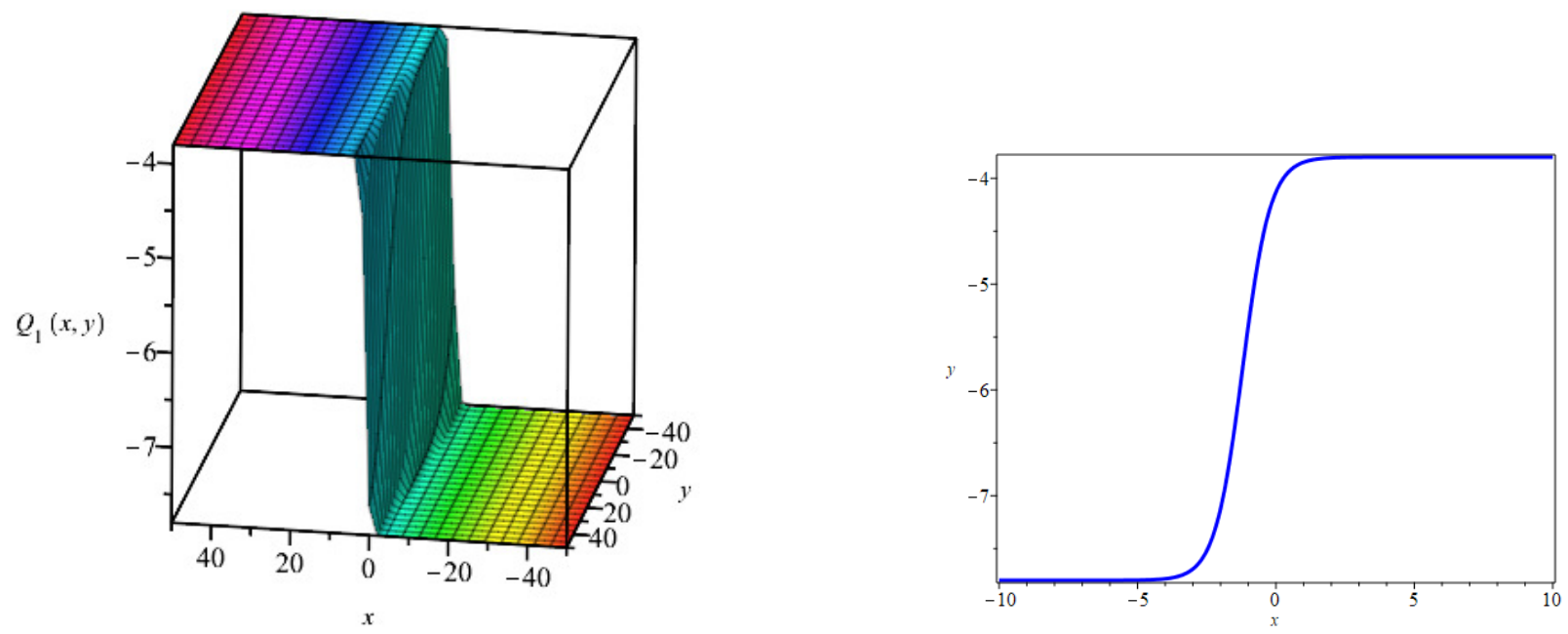

Figure 3.1: $3 \mathrm{D}$ dimensional surface and $2 \mathrm{D}$ graphs of solution of Eq. (3.3) where $y=1, z=1, t=1, a=2, a_{0}=0.2, b=-0.1, d=0.01, s=0.01, \beta=-0.1$, and $r=1$.

Case 1: $k=\frac{b^{3} \alpha}{a^{3}}, d=\frac{4 a^{2} b-b r-2 c \beta}{s}, b_{1}=-10 a, a_{1}=0$.

$$
\psi_{2}(\varsigma)=a_{0}-\frac{10 a \sin (\varsigma)}{\cos (\varsigma)+2 \sin (\varsigma)}
$$

So, we have a trigonometric type solution as below

$$
Q_{2}(x, y, z, t)=a_{0}+\frac{10 a \sin \left(c t-a x-b y-\frac{z\left(4 a^{2} b-b r-2 c \beta\right)}{s}\right)}{\cos \left(c t-a x-b y-\frac{z\left(4 a^{2} b-b r-2 c \beta\right)}{s}\right)-2 \sin \left(c t-a x-b y-\frac{z\left(4 a^{2} b-b r-2 c \beta\right)}{s}\right)} .
$$
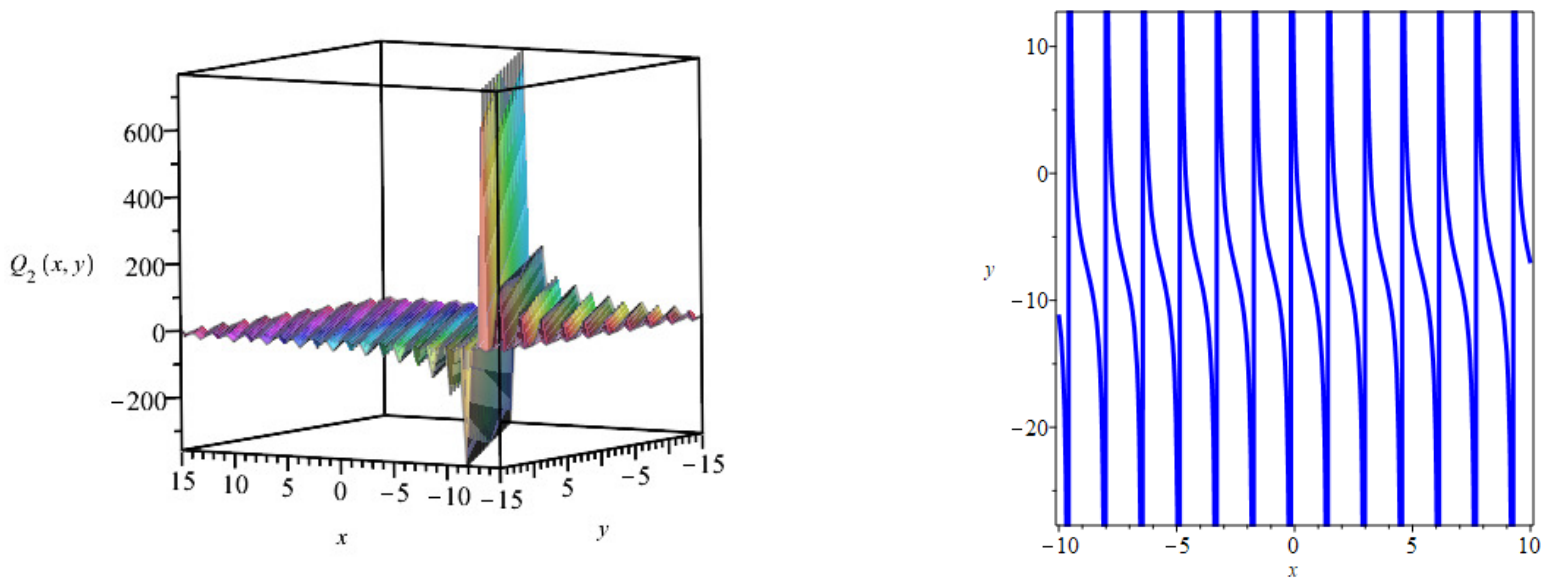

Figure 3.2: 3D dimensional surface and 2D graphs of solution of Eq. (3.4) for values of $\alpha=0.2, a=2, c=3, b=1, r=2, \beta=0.3, s=3 / 2, a_{0}=0.5, t=$ $1, z=1$, and $y=1$.

Family 3: Using $\gamma=\{2,0,1,1\}, \lambda=\{-1,0,1,-1\}$ in Eq. (2.5), so it takes the following form

$$
\phi(\varsigma)=\frac{\cosh (\varsigma)-\sinh (\varsigma)}{\cosh (\varsigma)} .
$$

Case 1: Plugging these results in with Eq. (3.5) into Eq. (3.2), we get

$$
\psi_{3}(\varsigma)=a_{0}-2 \operatorname{asech}(\varsigma)(\cosh (\varsigma)-\sinh (\varsigma)) .
$$

Then, one may have a solution of hyperbolic type as follows

$$
Q_{3}(x, y, z, t)=a_{0}-2 a \operatorname{sech}(c t-a x-b y-d z)(\cosh (c t-a x-b y-d z)+\sinh (c t-a x-b y-d z)) .
$$



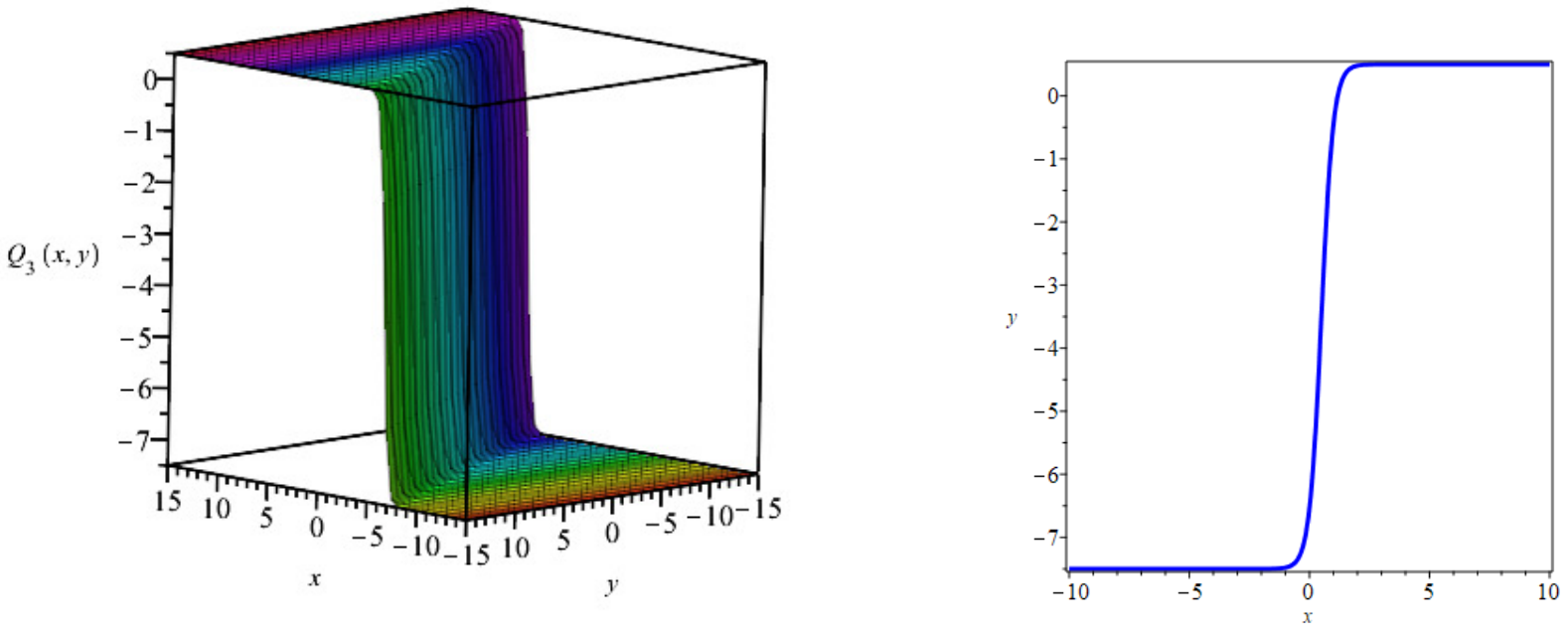

Figure 3.3: 3D dimensional surface and 2D graphs of solution of Eq. (3.6) for $\alpha=0.2, a=2, c=3, b=1, a_{0}=0.5, t=1, z=1, d=1$, and $y=1$.

Family 4: Using $\gamma=\{-1,1,1,1\}, \lambda=\{1,-1,1,-1\}$, then Eq. (2.5) turns to

$$
\phi(\varsigma)=\frac{-\sinh (\varsigma)}{\cosh (\varsigma)}
$$

Case 1: $b_{1}=-2 a, s=-\frac{16 a^{2} b+b r+2 c \beta}{d}, a_{1}=-2 a, \alpha=\frac{a^{3} k}{b^{3}}$.

Using the above values with Eq. (3.7) in Eq. (3.2), we have

$$
\psi_{4}(\varsigma)=a_{0}+2 a \operatorname{coth}(\varsigma)+2 a \tanh (\varsigma) .
$$

Therefore, we have

$$
Q_{4}(x, y, z, t)=a_{0}-2 a \operatorname{coth}(c t-a x-b y-d z)-2 a \tanh (c t-a x-b y-d z)
$$
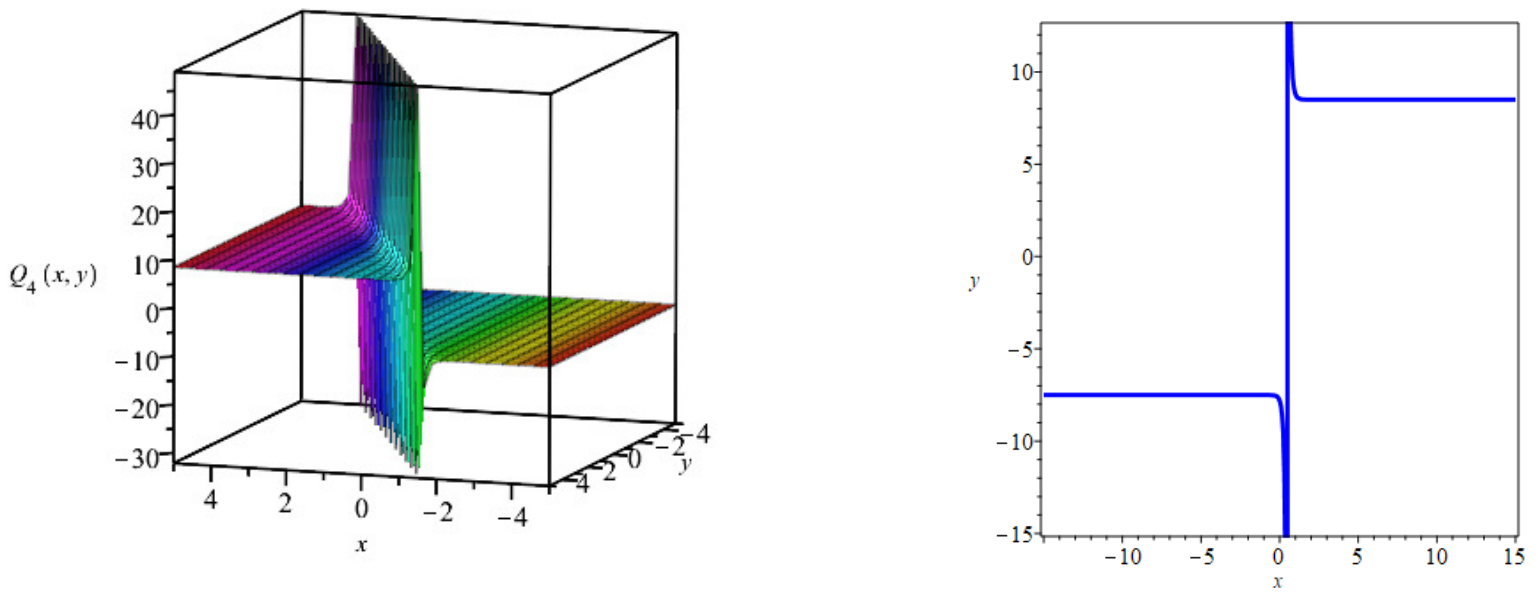

Figure 3.4: $3 \mathrm{D}$ dimensional surface and $2 \mathrm{D}$ graphs of solution of Eq. (3.8) for the values of $\alpha=0.2, a=2, c=3, b=1, a_{0}=0.5, t=1, z=1, d=1$, and $y=1$.

Family 5: Using $\gamma=\{i,-i, 1,1\}, \lambda=\{i,-i, i,-i\}$ in Eq. (2.5), then we have

$$
\phi(\varsigma)=\frac{\sin (\varsigma)}{\cos (\varsigma)}
$$

Case 1: $b_{1}=-\frac{2 b \alpha^{1 / 3}}{k^{1 / 3}}, a=\frac{b \alpha^{1 / 3}}{k^{1 / 3}}, \beta=-\frac{b r+d s-\frac{16 b^{3} \alpha^{2 / 3}}{k^{2 / 3}}}{2 c}, a_{1}=\frac{2 b \alpha^{1 / 3}}{k^{1 / 3}}$. Inserting these values with Eq. (3.9) in Eq. (3.2), we have

$$
\psi(\varsigma)=a_{0}+\frac{2 b \alpha^{1 / 3} \cot (\varsigma)}{k^{1 / 3}}-\frac{2 b \alpha^{1 / 3} \tan (\varsigma)}{k^{1 / 3}} .
$$


So, we have

$$
Q_{5}(x, y, z, t)=a_{0}-\frac{2 b \alpha^{1 / 3} \cot \left(c t-b y-d z-\frac{b x \alpha^{1 / 3}}{k^{1 / 3}}\right)}{k^{1 / 3}}+\frac{2 b \alpha^{1 / 3} \tan \left(c t-b y-d z-\frac{b x \alpha^{1 / 3}}{k^{1 / 3}}\right)}{k^{1 / 3}} .
$$
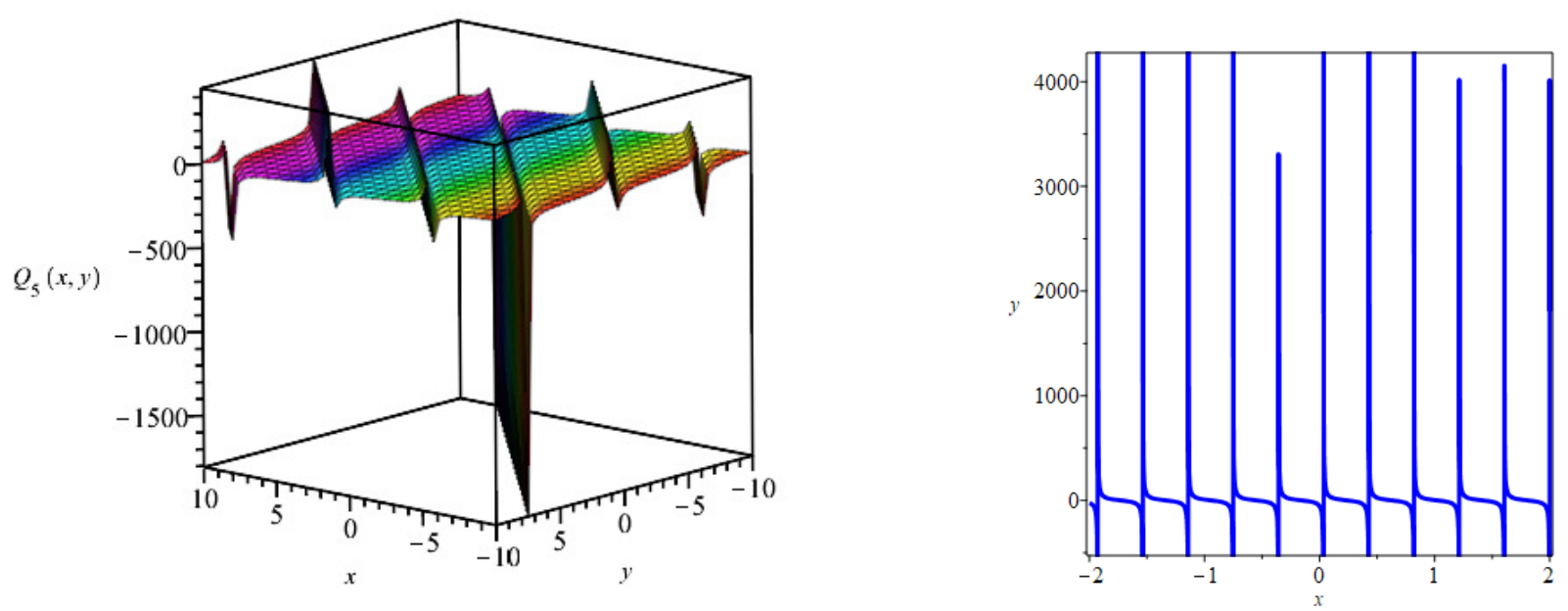

Figure 3.5: 3D dimensional surface and 2D graphs of solution of Eq. (3.10) where $\alpha=8, a=2, c=3, b=4, a_{0}=0.5, t=1, z=1, d=2, k=8$, and $y=1$.

Family 6: Using $\gamma=\{-1,0,1,1\}, \lambda=\{0,0,0,1\}$, then Eq. (2.5) turns to

$$
\phi(\varsigma)=-\frac{1}{1+\mathrm{e}^{\varsigma}} .
$$

Case 1: $b_{1}=0, \alpha=\frac{a^{3} k}{b^{3}}, s=-\frac{b\left(a^{2}+r\right)+2 c \beta}{d}, a_{1}=2 a$. Inserting the above values with Eq. (3.11) to Eq. (3.2), we have

$$
\phi(\varsigma)=a_{0}-\frac{2 a}{1+\mathrm{e}^{\varsigma}} .
$$

Therefore,

$$
Q_{6}(x, y, z, t)=a_{0}-\frac{2 a}{1+\mathrm{e}^{-c t+a x+b y+d z}}
$$
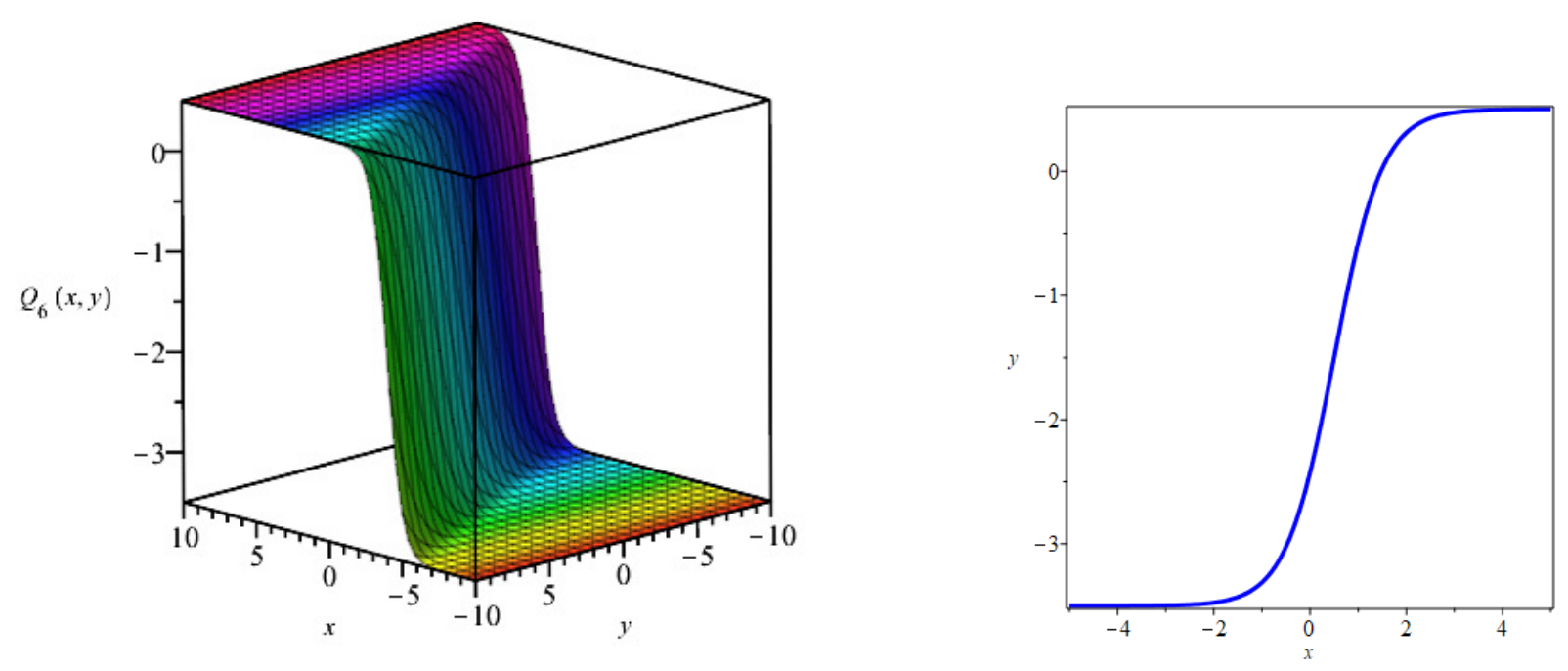

Figure 3.6: 3D dimensional surface and 2D graphs of solution of Eq. (3.12) using $a=2, c=3, b=1, a_{0}=0.5, t=1, z=1, d=1$, and $y=1$.

Family 7: Using $\gamma=\{1+i, 1-i, 1,1\}, \lambda=\{i,-i, i,-i\}$ Eq. (2.5) turns to

$$
\phi(\varsigma)=\frac{-\sin (\varsigma)+\cos (\varsigma)}{\cos (\varsigma)}
$$


Case 1: $b_{1}=-\frac{4 b \alpha^{1 / 3}}{k^{1 / 3}}, a=\frac{b \alpha^{1 / 3}}{k^{1 / 3}}, a_{1}=0, s=\frac{b r-\frac{4 b^{3} \alpha^{2 / 3}}{k^{2 / 3}}+2 c \beta}{d}$. Putting these values with Eq. (3.16) into Eq. (3.3), we have

$$
\psi_{7}(\varsigma)=a_{0}-\frac{4 b \alpha^{1 / 3} \cos (\varsigma)}{k^{1 / 3}(\cos (\varsigma)-\sin (\varsigma))} .
$$

So, we have

$$
Q_{7}(x, y, z, t)=a_{0}-\frac{4 b \alpha^{1 / 3} \cos \left(c t-b y-d z-\frac{b x \alpha^{1 / 3}}{k^{1 / 3}}\right)}{k^{1 / 3}\left(\cos \left(c t-b y-d z-\frac{b x \alpha^{1 / 3}}{k^{1 / 3}}\right)+\sin \left(c t-b y-d z-\frac{b x \alpha^{1 / 3}}{k^{1 / 3}}\right)\right)} .
$$
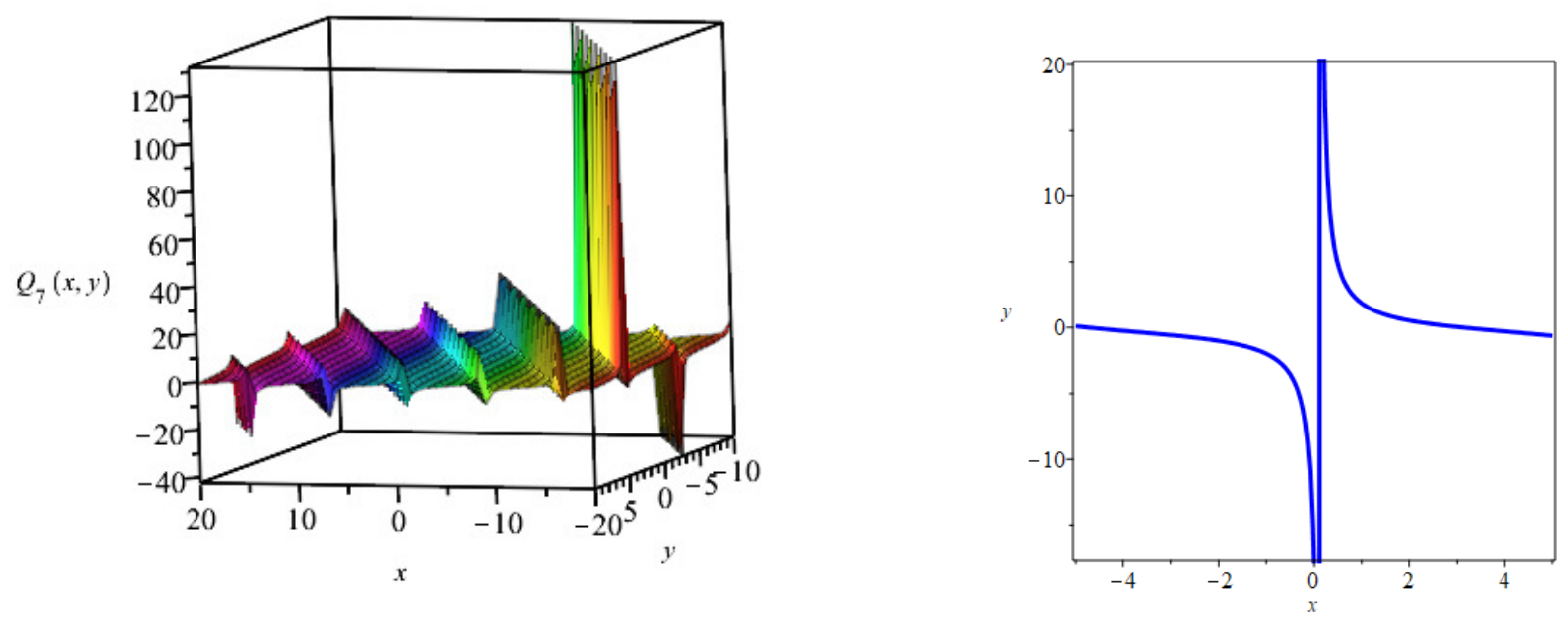

Figure 3.7: : 3D dimensional surface and 2D graphs of solution of Eq. (3.14) using $\alpha=8, a=2, c=3, b=0.4, a_{0}=0.5,, d=0.2, k=8, t=1, z=1$, and $y=1$.

\section{Conclusions}

In this study, we have investigated the (3+1)-dimensional Date-Jimbo-Kashiwara-Miwa equation via using the generalized exponential rational function method, which is the most power method to study NPDEs. The feature of this method is that we can find abundant different solutions that are dependent on the parameters involved in it. As a result, we constructed some new exact solutions in the type of exponential, trigonometric and hyperbolic solutions. The 3D surfaces and 2D graphs of all obtained solutions are plotted. All constructed solutions satisfy the original equation.

\section{Acknowledgments}

The author would like to express their sincere thanks to the editor and the anonymous reviewers for their helpful comments and suggestions.

\section{Funding}

There is no funding for this work.

\section{Availability of data and materials}

Not applicable.

\section{Competing interests}

The author declare that she has no competing interests.

\section{Authors contributions}

The author read and approved the final manuscript. 


\section{References}

[1] S. Kumar, H. Almusawa, I. Hamid, MA Akbar, MA Abdou, Abundant analytical soliton solutions and Evolutionary behaviors of various wave profiles to the Chaffee-Infante equation with gas diffusion in a homogeneous medium, Results Phys., (2021), Article ID 104866.

[2] K. K. ALi, R. Yilmazer, H. M. Baskonus, H. Bulut, Modulation instability analysis and analytical solutions to the system of equations for the ion sound and Langmuir waves, Phys. Scr., 95 (2020), Article ID 065602.

[3] H. Dutta, H. Günerhan, K. K. Ali, R. Yilmazer, Exact Soliton Solutions to the Cubic-Quartic Non-linear Schrödinger Equation With Conformable Derivative, Frontiers in Physics 8 (2020).

[4] W. H. Zhu, L. G. Liu, Stripe solitons and lump solutions to a generalized (3+ 1)-dimensional B-type Kadomtsev-Petviashvili equation with variable coefficients in fluid dynamics, J. Math. Anal. Appl., 502 (2021), Article ID 125198.

[5] J. Manafian, O. A. Ilhan, K. K. Ali, S. Abid, Cross-kink wave solutions and semi-inverse variational method for (3+ 1)-dimensional potential-YTSF equation, East Asian J. Appl. Math., 10 (2020), 549-65.

[6] H. F. Ismael, H. Bulut, H. M. Baskonus, W. Gao, Dynamical behaviors to the coupled Schrödinger-Boussinesq system with the beta derivative, AIMS Math., 6 (2021), 7909-28.

[7] K. K. Ali, R. Yilmazer, H. Bulut, T. Aktürk, M. S. Osman, Abundant exact solutions to the strain wave equation in micro-structured solids, Modern Phys. Lett. B, 35 (2021), Article ID 2150439.

[8] H. F. Ismael, A. Seadawy, H. Bulut, Multiple soliton, fusion, breather, lump, mixed kink-lump and periodic solutions to the extended shallow water wave model in (2+ 1)-dimensions, Modern Phys. Lett. B, 35 (2021), Article ID 2150138.

[9] J. G. Liu, W. H. Zhu, Y. He, Variable-coefficient symbolic computation approach for finding multiple rogue wave solutions of nonlinear system with variable coefficients, Z. Angew. Math. Phys., 72 (2021), 1-12.

[10] W. X. Ma, T. Huang, Y. Zhang, A multiple exp-function method for nonlinear differential equations and its application, Phys. Scr., 82 (2010), Article ID

[11] H. M. Baskonus, New acoustic wave behaviors to the Davey-Stewartson equation with power-law nonlinearity arising in fluid dynamics, Nonlinear Dynam., (2016).

[12] Y. X. Li, E. Celik, J. L. Guirao, T. Saeed, H. M. Baskonus, On the modulation instability analysis and deeper properties of the cubic nonlinear Schrödinger's equation with repulsive $\delta$-potential, Results Phys., 25 (2021)., Aertcle ID 104303.

[13] S. T. Demiray, H. Bulut, E. Celik, Soliton solutions of Wu-Zhang system by generalized Kudryashov method, AIP Conference Proceedings (2037), (2018), (020025).

[14] H. F. Ismael, H. Bulut, H. M. Baskonus, Optical soliton solutions to the Fokas-Lenells equation via sine-Gordon expansion method and ( $m+(G$ / $G))$ expansion method, Pramana, 94 (2020), 1-9.

[15] E. Celik, H. Bulut, H. M. Baskonus, Novel features of the nonlinear model arising in nano-ionic currents throughout microtubules, Indian J. Phys., 92 (2018), 1137-1143.

[16] J.P. Fang, Q.B. Ren, C.L. Zheng, New exact solutions and fractal localized structures for the (2+1)-dimensional Boiti-Leon-Pempinelli system. Z. Naturforsch, 60 (2005), 245-251.

[17] M. A. Dokuyucu, E. Celik, Analyzing a novel coronavirus model (COVID-19) in the sense of caputo-fabrizio fractional operator. Comput. Appl. Math., (2021), 49-69.

[18] H.F. Ismael, M. A. S. Murad, H. Bulut, Various exact wave solutions for KdV equation with time-variable coefficients, J. Ocean Eng. Sci., (2021).

[19] H. F. Ismael, H. Bulut, Nonlinear dynamics of (2+1)-dimensional Bogoyavlenskii-Schieff equation arising in plasma physics, Math. Methods Appl. Sci., 44(13) (2021), 10321-10330.

[20] H. F. Ismael, H. Bulut, Multi soliton solutions, M-lump waves and mixed soliton-lump solutions to the awada-Kotera equation in (2+ 1)-dimensions, Chinese J. Phys., 71 (2021), 54-61.

[21] F. Dusunceli, E. Celik, M. Askin, H. Bulut, New exact solutions for the doubly dispersive equation using the improved Bernoulli sub-equation function method, Indian J. Phys., 95 (2021) 309-314.

[22] X. Guan, W. Liu, Q. Zhou, A. Biswas, Darboux transformation and analytic solutions for a generalized super-NLS-mKdV equation, Nonlinear Dyn., 98 (2019), 1491-1500.

[23] K. K. Ali, R. Yilmazer, M. S. Osman, Extended Calogero-Bogoyavlenskii-Schff equation and its dynamical behaviors, Phys Scr., (2021).

[24] E. Date, M. Jimbo, M. Kashiwara, T. Miwa, Transformation groups for soliton equations: IV. A new hierarchy of soliton equations of KP-type, Phys. D., 4 (1982), 343-65.

[25] A. M. Wazwaz, A (2+1)-dimensional time-dependent Date-Jimbo-Kashiwara-Miwa equation: Painlevé integrability and multiple soliton solutions, Comput. Math. Appl., $79(2020), 1145-9$.

[26] H. F. Ismael, A. Seadawy, H. Bulut, Rational solutions, and the interaction solutions to the (2+ 1)-dimensional time-dependent Date-Jimbo-Kashiwara-Miwa equation, Int. J. Comput. Math., 9812 (2021), 2369-2377.

[27] A. W. Wazwaz, New (3+1)-dimensional Date-Jimbo-Kashiwara-Miwa equations with constant and time-dependent coefficients: Painlevé integrability, Phys. Lett. A, 384 (2020), 126787.

[28] H. Almusawa, K. K. Ali, A. M. Wazwaz, M. S. Mehanna, D. Baleanu, M.S. Osman, Protracted study on a real physical phenomenon generated by media inhomogeneities, Results Phys., 31 (2021), Article ID 104933.

[29] B. Ghanbari, C. K. Kuo, New exact wave solutions of the variable-coefficient (1+ 1)-dimensional Benjamin-Bona-Mahony and (2+ 1)-dimensional asymmetric Nizhnik-Novikov-Veselov equations via the generalized exponential rational function method, Eur. Phys. J. Plus, 134 (2019), 1-13.

[30] B. Ghanbari, Abundant soliton solutions for the Hirota-Maccari equation via the generalized exponential rational function method, Modern Phys. Lett. B, 33(9) (2019), 1950106.

[31] B. Ghanbari, M. Inc, A new generalized exponential rational function method to find exact special solutions for the resonance nonlinear Schrödinger equation Eur. Phys. J. Plus, 133(4) (2018).

[32] K. K. Ali, H. Dutta, R. Yilmazer, S. Noeiaghdam, On the new wave behaviors of the Gilson-Pickering equation, Front Phys., 8(2020). 